\title{
In vitro screening of radioprotective properties in the novel glucosylated flavonoids
}

\author{
HAO YU ${ }^{1,2}$, JEREMY S. HASKINS ${ }^{1}$, CATHY SU ${ }^{1}$, ALLISON ALLUM ${ }^{1}$, ALEXIS H. HASKINS ${ }^{1}$, \\ VICTORIA A. SALINAS ${ }^{1}$, SHIGEAKI SUNADA ${ }^{2}$, TAISUKE INOUE ${ }^{2}$, YASUSHI AIZAWA ${ }^{3,4}$, \\ MITSURU UESAKA ${ }^{2,3}$ and TAKAMITSU A. KATO ${ }^{1}$ \\ ${ }^{1}$ Department of Environmental and Radiological Health Sciences, Colorado State University, Fort Collins, \\ CO 80523-1618, USA; Departments of ${ }^{2}$ Nuclear Engineering and Management, and ${ }^{3}$ Bioengineering, \\ School of Engineering, The University of Tokyo, Tokyo 113-8656; ${ }^{4}$ Research and Development Group, \\ Toyo Sugar Refining Co., Ltd., Yoto Bldg., Tokyo 103-0046, Japan
}

Received March 25, 2016; Accepted August 22, 2016

DOI: $10.3892 /$ ijmm.2016.2764

\begin{abstract}
Novel glucosyl flavonoids are developed by the addition of glucose to naturally occurring flavonoids. Flavonoids are known antioxidants that possess radioprotective properties. In order to investigate the radioprotective properties of novel glucosyl flavonoids, in vitro DNA double-strand breaks (DSBs) analysis was carried out. In the present study, Quercetin, Naringenin, and Hesperetin groups of flavonoids included in the natural and novel glucosyl 13 flavonoids were investigated. Flavonoids were mixed with Lambda DNA, and subsequently exposed to gamma-rays. Furthermore, DNA DSB yields were visualized by gel electrophoresis. Quercetin derivatives displayed reduced DNA DSB formation at $10 \mu \mathrm{M}$. At a high concentration, the majority of flavonoids displayed radioprotective properties as a reduction of DSB yields. Suppression of DSB formation was confirmed via the molecular combing assay for Quercetin, and three monoglucosyl flavonoids. Glucosylation showed positive effects for radioprotection and monoglucosyl-Rutin showed superior radioprotective properties when compared to monoglucosyl-Naringin and Hesperidin. In addition, Quercetin derivatives had greater total antioxidant capacities and DPPH radical scavenging ability than other flavonoid groups. Since Quercetin, Isoquercetin, and Rutin display poor water solubility, monoglucosyl-Rutin, maltooligosyl-Isoquercetin, and maltooligosyl-Rutin may be better radioprotective agents and easily bioavailable with increased water solubility.
\end{abstract}

Correspondence to: Professor Takamitsu A. Kato, Department of Environmental and Radiological Health Sciences, Colorado State University, 1618 Campus Delivery, Fort Collins, CO 80523-1618, USA

Email: takamitsu.kato@colostate.edu

Key words: flavonoids, glucosylated flavonoids, DNA double-strand breaks

\section{Introduction}

A noteworthy property of ionizing radiation is its ability to produce highly reactive free radicals [hydroxyl radical $(\bullet \mathrm{OH})](1)$. Free radicals, whether formed by indirect or direct action, eventually damage biologically important materials such as DNA and lead to radiation-induced damage formation (2). This damage may be single-strand breaks (SSBs), base damage or the most molecularly deleterious double-strand breaks (DSBs). Effectively, the harmful damage produced by strong oxidation can damage the body's tissues and cells, thereby causing chronic diseases including cancer and accelerated aging $(3,4)$.

As with all biological systems there are methods of protection against harmful exogenous genotoxic mutagens $(5,6)$. Flavonoids natural occur in plants and other leafy organisms $(7,8)$. Flavonoids are also commonly regarded as antioxidant (9). New evidence suggests that these antioxidant properties may help plants protect themselves from harmful UV and ionizing radiation by absorbing harmful free radicals $(10,11)$.

Moreover, if a flavonoid is void of a glucose entity, then they are referred to as aglycons. Naturally occurring flavonoids hold none or few glucosyl residues coinciding with an aglycon (7). Additionally, novel flavonoids are synthesized by glucosylating the original glucosyl flavonoids. Glucosylation changes many properties of a flavonoid and specifically enhances their solubility in water $(12,13)$. Our previous study supports that glucosylation reduces cellular toxicity and genotoxicity in tissue culture systems (14). Furthermore, we suggested this may be due to a reduction of bioavailability in cells by glucosylation or a reduction in chemical properties, such as an inhibitory effect of PARP (15).

Previous findings suggest that a select few flavonoids and glucosyl flavonoids possess radioprotective properties (16). These include glucosyl chemicals such as the glucosylation of an ascorbic acid product, and ascorbic acid-2-glucoside, which did not alter the radioprotective properties of ascorbic acid $(17,18)$. However, the effect that glucosylation has on the radioprotective properties of flavonoids remains to be determined. 
Among radiation-induced DNA damage, DNA DSBs contribute the most towards radiation-induced cell death. DNA DSBs can be measured by an array of assay systems such as neutral elution $(19,20)$, gel electrophoresis $(21,22)$, and immunohistochemistry $(22,23)$. For the present study 13 natural and novel synthesized flavonoids were used. Naked Lambda DNA was run through gel electrophoresis to visualize the suppression of DNA DSB formation by the chemicals. In the present study, we investigated whether glucosylation affects the antioxidant and radical scavenging properties of flavonoids. The radioprotective effects of novel glucosyl flavonoids were constructed visually via a molecular combing technique (24).

\section{Materials and methods}

Chemicals. The flavonoids were obtained from the Toyo Sugar Refining Co., Ltd. (Tokyo, Japan). Fig. 1a shows the chemical structures of natural and novel synthetic flavonoids used in the present study. The Quercetin group treatment consisted of Quercetin (302.23 g/mol), Isoquercetin (464.58 g/mol), Rutin (610.51 g/mol), monoglucosyl (MG)-Rutin (772.5 g/mol), and maltooligosyl (MO)-Isoquercetin (914.38 g/mol), MO-Rutin (1104.4 g/mol). The Naringenin group treatment consisted of Naringenin $(272.257 \mathrm{~g} / \mathrm{mol})$, Naringin $(580.54 \mathrm{~g} / \mathrm{mol})$, MG-Naringin $(742.54 \mathrm{~g} / \mathrm{mol})$ and MO-Prunin $(922.54 \mathrm{~g} / \mathrm{mol})$. The Hesperetin group treatment consisted of Hesperetin (302.28 g/mol), Hesperidin $(610.57 \mathrm{~g} / \mathrm{mol})$ and MG-Heperidin $(610.57 \mathrm{~g} / \mathrm{mol})$. Flavonoids were dissolved in double-distilled water. Their $\mathrm{pH}$ values at $100 \mu \mathrm{M}$ solution showed slightly acidic to neutral, ranging from 6.0 to 6.9. Turbidity of solution was measured by VersaMax ELISA microplate reader with SoftMax Pro (Molecular Devices, LLC, Sunnyvale, CA, USA) software at $660 \mathrm{~nm}$. Lambda DNA in Tris-EDTA was purchased from Nippon Gene Co., Ltd. (Tokyo, Japan). 2,2-Diphenyl-1-picrylhydrazyl (DPPH) was purchased from Sigma-Aldrich (St. Louis, MO, USA) and freshly prepared for each experiment in ethanol.

Irradiation. For gamma-ray irradiation, a J.L. Shepherd Model Mark I-68 nominal $6000 \mathrm{Ci}{ }^{137} \mathrm{Cs}$ irradiator (J.L. Shepherd and Associates, San Fernando, CA, USA) was used at room temperature $\left(20^{\circ} \mathrm{C}\right)$. The dose rate was $3.9 \mathrm{~Gy} / \mathrm{min}$.

Electrophoresis and DNA DSBs. Two microliters of Lambda DNA $(12 \mu \mathrm{g} / \mu \mathrm{l})$ was mixed with $18 \mu \mathrm{l}$ of each concentration $(0,10,100 \mu \mathrm{M})$ of flavonoids. Lambda DNA HindIII digest $(0.2 \mu \mathrm{l})$ was used as a marker. A total of $20 \mu \mathrm{l}$ of sample was exposed to gamma-rays, and run through electrophoresis after mixing DNA with $4 \mu \mathrm{l}$ of $6 \mathrm{X}$ DNA loading dye. The Lambda DNA and the marker were immediately immersed in a $60^{\circ} \mathrm{C}$ water bath for $5 \mathrm{~min}$ to allow for the denaturion of DNA and the samples were placed on ice for $3 \mathrm{~min}$. The gel comprised $0.5 \mathrm{~g}$ agarose with $50 \mathrm{ml}$ of $1 \mathrm{X}$ TAE buffer. Electrophoresis was carried out at $100 \mathrm{~V}(7.63 \mathrm{~V} / \mathrm{cm})$ for $1 \mathrm{~h}$ in $1 \mathrm{X}$ TAE buffer. After electrophoresis, the DNA was stained in ethidium bromide solution for at least $3 \mathrm{~h}$. The gel was washed with $1 \mathrm{X}$ TAE buffer for $1 \mathrm{~h}$. Gel images were obtained with the Molecular Imager Gel Doc XR system with Image Lab software (Bio-Rad Laboratories, Inc., Hercules, CA, USA).
Single molecule observation. To evaluate the induction of DNA DSBs visually, single molecule observation was conducted using a molecular combing method (24). Before irradiation, the $60 \times 24 \mathrm{~mm}$ cover glass (no. 24601; Matsunami Glass Ind., Inc., Osaka, Japan) was pretreated with $30 \% \mathrm{H}_{2} \mathrm{O}_{2}$ at $4^{\circ} \mathrm{C}$ for at least $4 \mathrm{~h}$, and a coverslip was washed with distilled water and $100 \%$ ethanol. After irradiation, $100 \mu \mathrm{l}$ samples were stained with $0.02 \mu 11 \mathrm{mM}$ YOYO-1 (Thermo Fisher Scientific, Inc., Waltham, MA, USA), a fluorescent dye reagent mixed with $0.2 \mu \mathrm{l} 14.3 \mathrm{M}$ 2-mercaptoethanol and $1 \mu \mathrm{l}$ DMSO.

The cover glass was set at $45^{\circ}$ angles. A $50 \mu 1$ Lambda DNA solution was dropped onto the top end of the coverslip and allowed to run down to the bottom due to gravity. Additionally, the coverslip was placed on the glass slide without a mounting agent. Images of Lambda DNA were captured using a Zeiss Axioplan microscope (Carl Zeiss AG, Oberkochen, Germany) with Q-Imaging EXi Aqua CCD camera with QCapture Pro software (QImaging, Surrey, BC, Canada). The evaluation was performed by measuring the length of Lambda DNA using QCapture Pro software. At least 50 DNA samples were scored for three independent experiments.

Total antioxidant capacity (TAC). The antioxidant activity of each chemical was measured by a Total Antioxidant Capacity kit (Sigma-MAK187) according to the manufacturer's instructions. $\mathrm{Cu}^{2+}$ reagent was diluted 50 -fold with assay diluent. Five microliters of each concentration of flavonoid were diluted at a 1:1 ratio with 50 -fold diluted $\mathrm{Cu}^{2+}$ reagent. The samples were mixed and incubated for $90 \mathrm{~min}$ at room temperature. The absorbance of each sample was measured at $570 \mathrm{~nm}$ with NanoDrop (Thermo Fisher Scientific, Inc.). The values obtained with double-distilled water were used as the control. Each data point was produced by mean of three replicates per experiment and three independent experiments were carried out.

DPPH antioxidant properties. DPPH analysis was performed as previously reported (25). One hundred microliters of $100 \mu \mathrm{M}$ DPPH, $80 \mu \mathrm{l}$ of ethanol and $20 \mu \mathrm{l}$ each concentration of flavonoid were mixed. The mixtures were agitated vigorously and allowed to stand at room temperature for $30 \mathrm{~min}$. Absorbance was measured at $517 \mathrm{~nm}$ using a VersaMax ELISA Microplate Reader. DPPH scavenging activity was calculated by absorbance of control minus absorbance of sample divided by absorbance of control (26). Each data point was produced by mean of triplicates per experiment and three independent experiments were carried out.

Statistical analysis. Statistical comparison of the mean values was performed using a t-test with GraphPad Prism 6 (GraphPad Software, Inc., La Jolla, CA, USA). P $<0.05$ was considered to indicate a statistically significant difference. Error bars indicate the standard error of the mean.

\section{Results}

Turbidity of each flavonoid at absorbance at $660 \mathrm{~nm}$. Our results indicated that natural flavonoids were water insoluble and with increased glucosylation this resulted in improved water solubility. In the Quercetin group, the natural flavonoids, Quercetin, Isoquercetin and Rutin had poor solubility in water, 
a

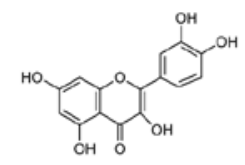

ii

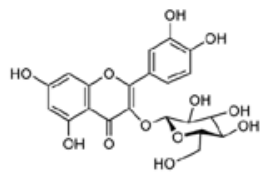

iii

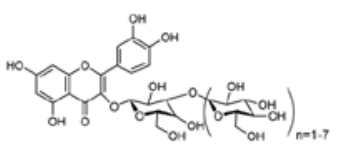

iv
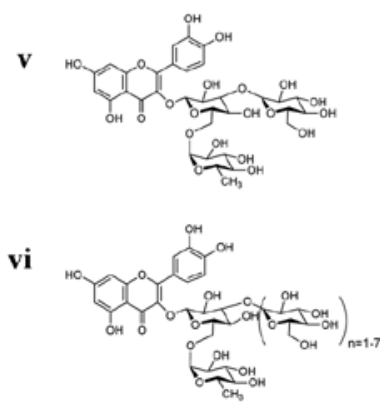

vii

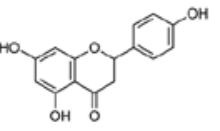

viii

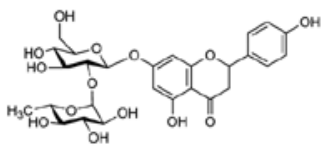

ix

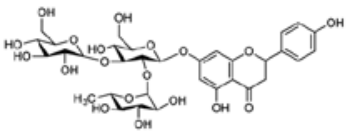

$\mathbf{x}$

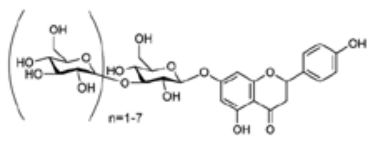

xi

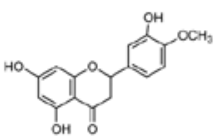

xii

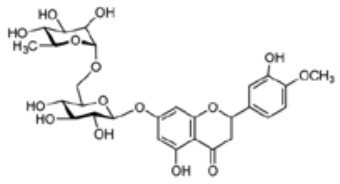

xiii

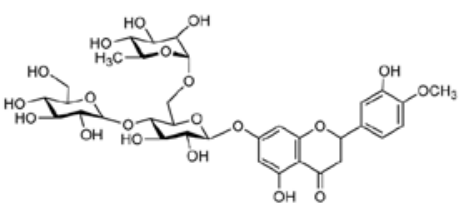

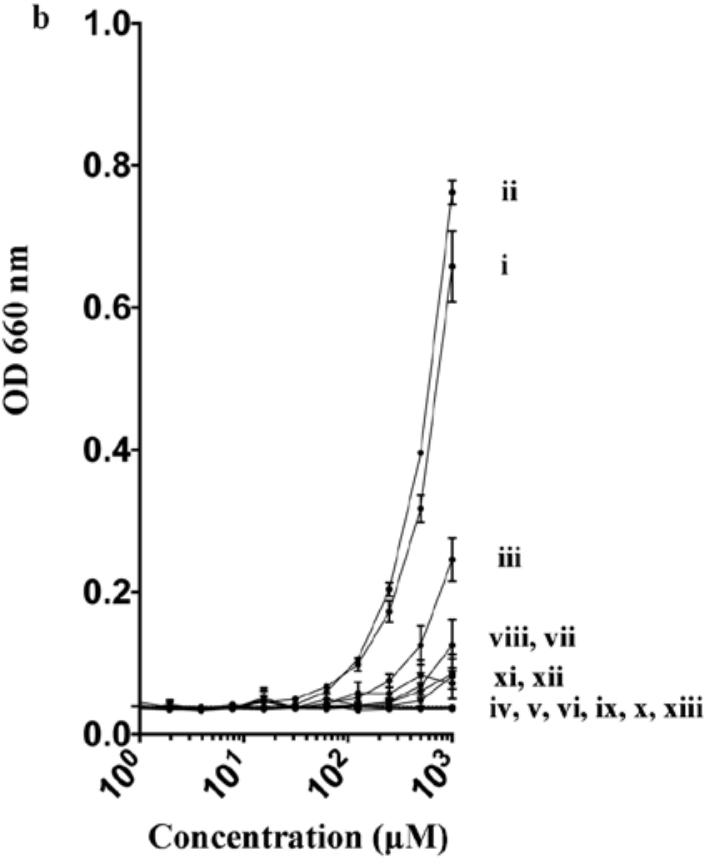

Figure 1. (a) Various chemical structures of 13 flavonoids in the present study. i) Quercetin, ii) Isoquercetin, iii) Rutin, iv) MG-Rutin, v) MO-Isoquercetin, vi) MO-Rutin, vii) Naringenin, viii) Naringin, ix) MG-Naringin, $x$ ) MO-Prunin, xi) Hesperetin, xii) Hesperidin, and xiii) MG-Hesperidin. (b) Turbidity of flavonoid solution measured at $660 \mathrm{~nm}$. Dashed line indicates background water value. Error bars indicate standard error of the means.

whereas monoglucosyl and maltooligosyl flavonoids had complete solubility, even at $1 \mathrm{mM}$ concentration (Fig. 1b).

Gel electrophoresis. In the gel electrophoresis assay, fragmented Lambda DNA migrated more and was observed as a smear. Fig. 2a shows the DNA DSBs were dose-dependent between 0 and 20 Gy for Lambda DNA in this assay. Fig. $2 b$ shows that flavonoids did not produce DSBs by themselves. Fig. $2 \mathrm{c}$ and $\mathrm{d}$ shows the DSB radioprotective abilities of flavonoids at 10 and $100 \mu \mathrm{M}$. For $10 \mu \mathrm{M}$, the Quercetin group, particularly Isoquercetin, MG-Rutin, and MO-Isoquercetin MO-Rutin, displayed the strongest radioprotective abilities. All the flavonoids displayed positive radioprotective abilities at concentrations of $100 \mu \mathrm{M}$, with the exception of Quercetin and Hesperetin. Therefore, glucosylation enhanced the radioprotective properties observed as a reduction of DSB formation.

Molecular combing. The flavonoids Quercetin (no glucosylation, water insoluble), MG-Rutin (glucosylated water soluble), MG-Naringin (glucosylated water soluble) and MG-Hesperidin (glucosylated water soluble) were tested for molecular combing. Fig. 3 shows the result of molecular combing. Each intact Lambda DNA molecule was observed as stretched DNA, and radiation-induced fragments of Lambda DNA were observed in a single molecule (Fig. $3 a$ and b). There was a dose-dependent DSB formation of 0 to $20 \mathrm{~Gy}$. Radiation (20 Gy) produced approximately 5 DSB/Lambda DNA. After exposure to $20 \mathrm{~Gy}$, the addition of $10 \mu \mathrm{M}$ MG-Rutin showed the strongest reduction in the frequency of DSB among the four flavonoids ( $\mathrm{p}<0.05$ ) (Fig. 3d), and the addition of $100 \mu \mathrm{M}$ of any MG-flavonoids greatly reduced the DSB frequency $(\mathrm{p}<0.005)$ but not Quercetin ( $\mathrm{p}=0.84$ ). MG-Naringin and MG-Hesperidin $(10 \mu \mathrm{M})$ did not show statistically significant radioprotection compared to the 20 Gy-irradiated control $(\mathrm{p}>0.1)$.

TAC. Fig. 4 shows the total antioxidant capacity of 13 flavonoids, which was measured at the optical density of $570 \mathrm{~nm}$. Quercetin group showed good TAC at each concentration compared with other flavonoid groups. The Quercetin groups also exhibited antioxidant properties similar to that of ascorbic acid at 10 and $100 \mu \mathrm{M}$, with the exception of $10 \mu \mathrm{M}$ Quercetin. The Naringenin and Hesperetin groups were poor antioxidants compared to the ascorbic acid at the tested concentrations. No clear correlation between glucosylation and total TAC was observed. 

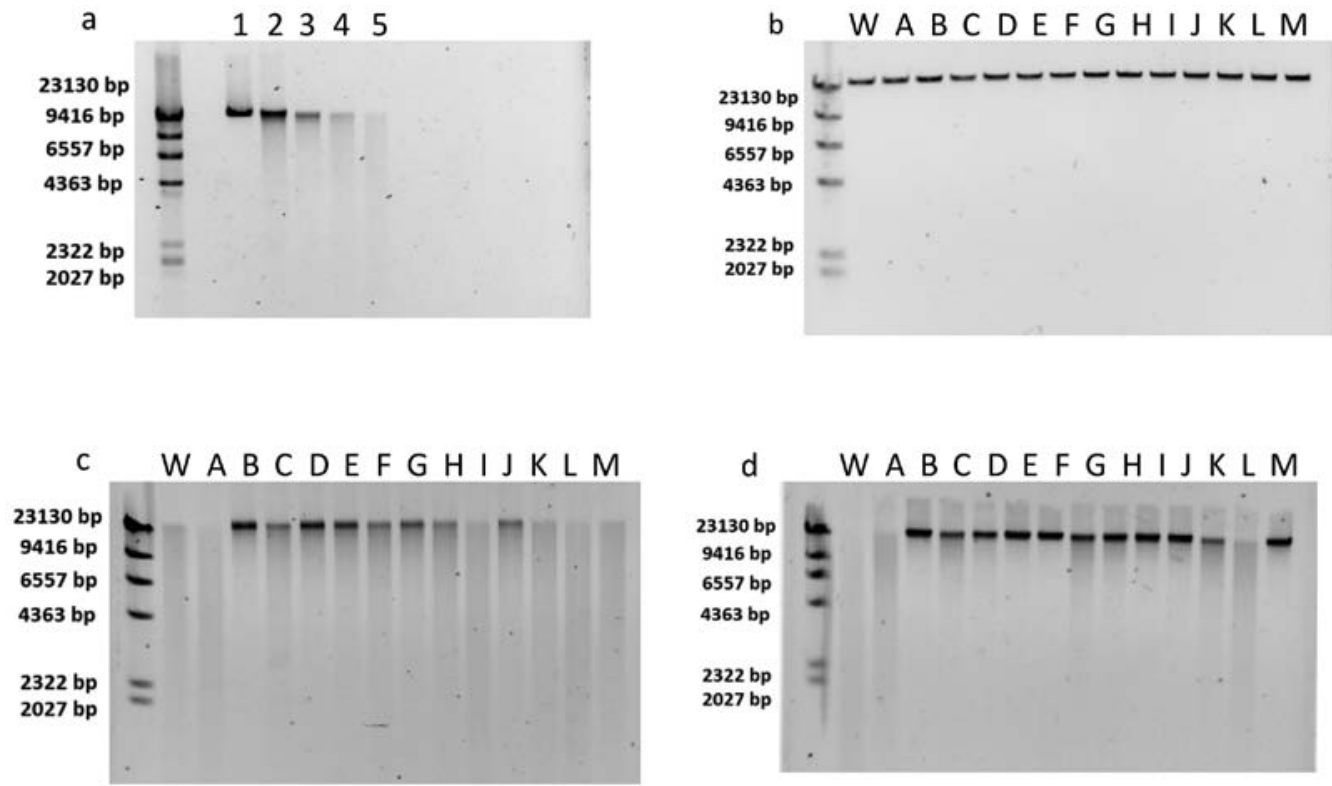

Figure 2. (a) Dose-dependent DNA double-strand breaks (DSBs). Lane 1, 0 Gy; lane 2, 5 Gy; lane 3, 10 Gy; lane 4, 15 Gy; and lane 5, 20 Gy. (b) Effect of flavonoids without irradiation. (c and d) Radioprotective effects of flavonoids at $10 \mu \mathrm{M}$ with $20 \mathrm{~Gy}$ and radioprotective effects of flavonoids at $100 \mu \mathrm{M}$ with 20 Gy. First left lane is Lambda DNA-HindIII digest ladder; lane W, is negative control with water; lane A, Quercetin; lane B, Isoquercetin; lane C, Rutin; lane D, MG-Rutin; lane E, MO-Isoquercetin; lane F, MO-Rutin; lane G, Naringenin; lane H, Naringin; lane I, MG-Naringin; lane J, MO-Prunin; lane K, Hesperetin; lane L, Hesperidin; and lane M, MG-Hesperidin.
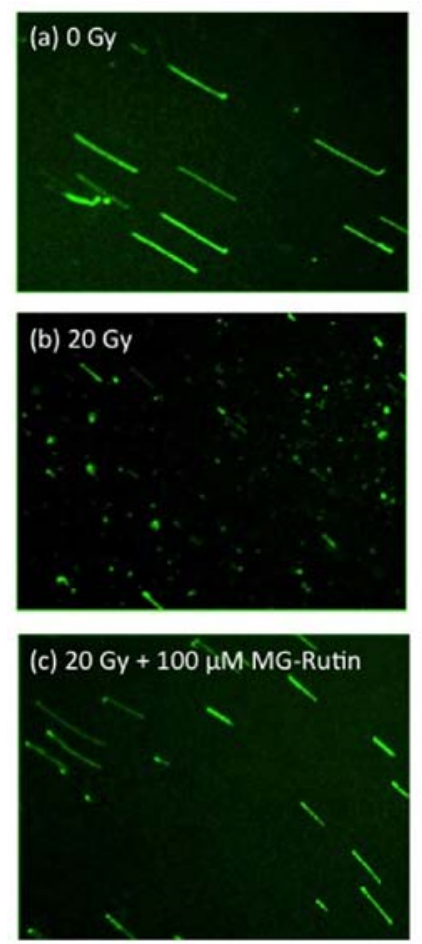

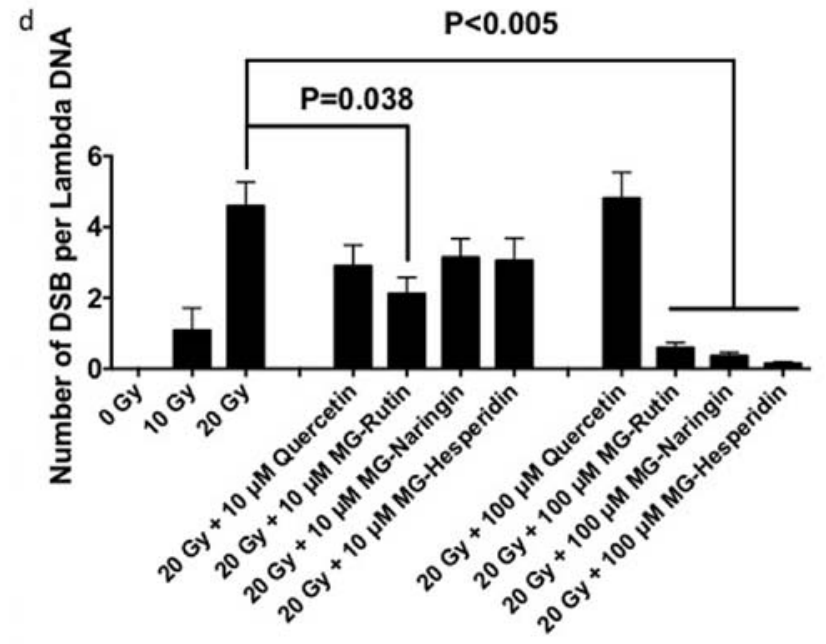

Figure 3. Molecular combing experiments. Images of molecular combing for (a) 0 Gy, (b) 20 Gy, and (c) 20 Gy $+100 \mu$ M MG-Rutin. (d) Number of DSB with radiation and flavonoids.

DPPH radical scavenging properties. DPPH is a stable free radical. Fig. 5 shows the result of DPPH radical scavenging properties. We used ascorbic acid as a control to compare the radical scavenging ability of 13 flavonoids. The Quercetin group showed much better radical scavenging ability than the other two groups. The result showed glucosylation slightly reduced free radical scavenging ability in the Quercetin group at 10 and $100 \mu \mathrm{M}$. The Naringenin and Hesperetin groups were poor radical scavengers at 10 and $100 \mu \mathrm{M}$.

\section{Discussion}

The results of the present study displayed that radioprotective properties for DSBs were dependent on flavonoid groups at 


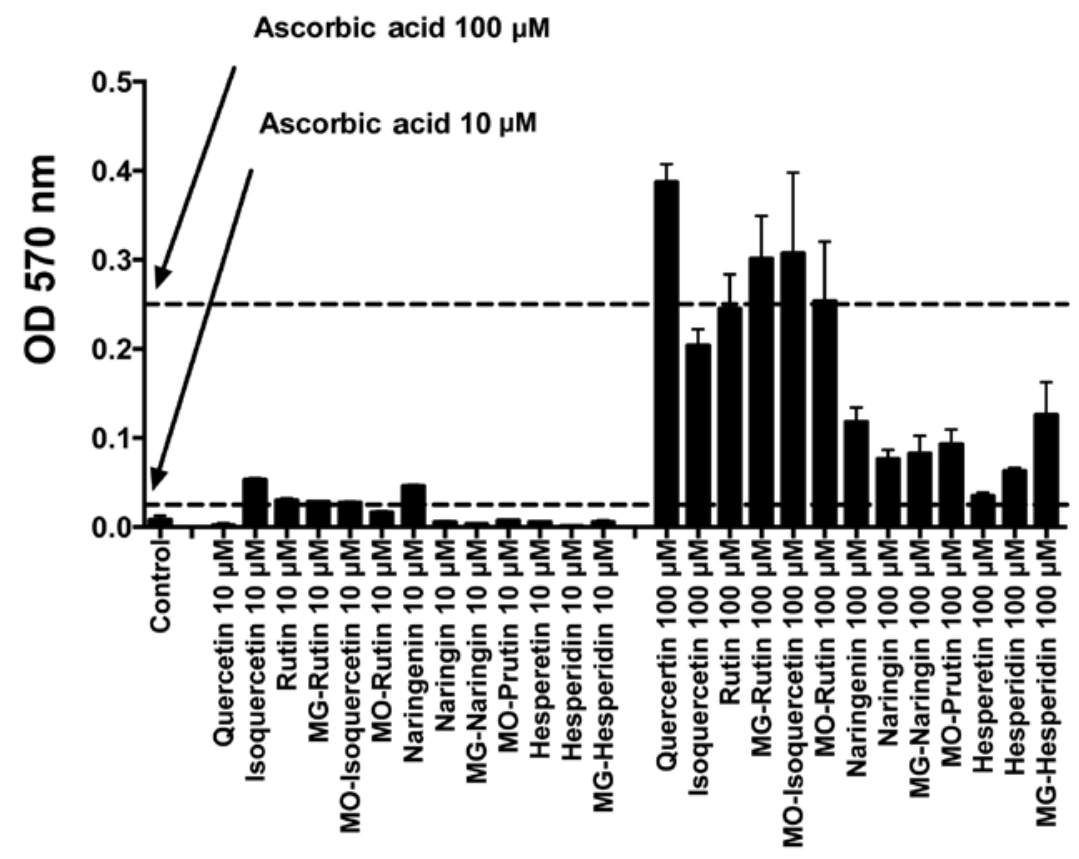

Figure 4. Total antioxidant capacity of 13 flavonoids. Dashed lines are 10 and $100 \mu \mathrm{M}$ ascorbic acid. Error bars indicate standard error of the means.

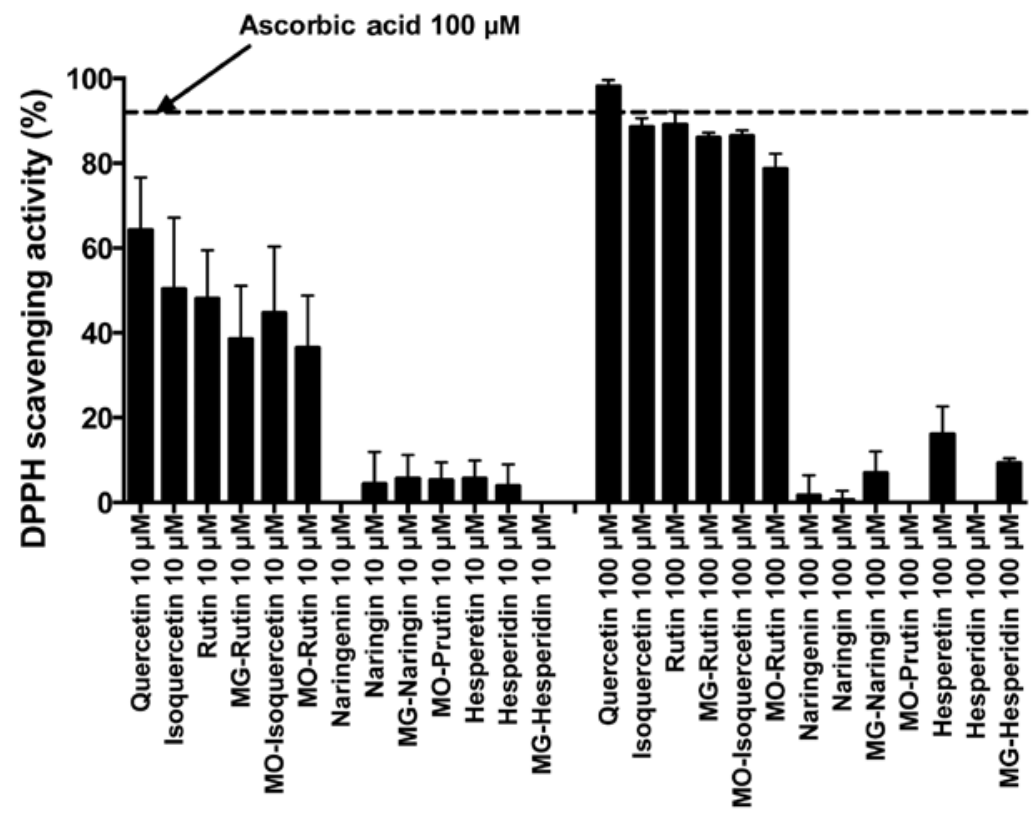

Figure 5. DPPH radical scavenging ability of 13 flavonoids. Dashed line is $100 \mu \mathrm{M}$ ascorbic acid. Error bars indicate standard error of the means.

a low concentration. All the Quercetin derivatives showed radioprotection after $20 \mathrm{~Gy}$, except natural Quercetin. On the other hand, the majority of flavonoids showed radioprotection at $100 \mu \mathrm{M}$. Therefore, an increased uptake of flavonoids may protect cells from DSB formation induced by radiation exposure. Better radioprotective properties in DSB reduction for the glucosylated Quercetin group were matched with total antioxidant capacity and radical scavenging ability. Although Quercetin showed strong radical scavenging and antioxidant properties, Quercetin did not show DSB reduction even at $100 \mu \mathrm{M}$. This may be associated with their poor water solubility or poor interaction with DNA molecules (27). Since Quercetin, Isoquercetin, and Rutin displayed poor water solubility, we suggested that MG-Rutin, MO-Isoquercetin, and MO-Rutin are better radioprotective agents and easily bioavailable with increased water solubility. Recently, enhanced solubility of Quercetin by forming composite particles with glucosyl flavonoids was reported (28). Enhanced solubility can be produced by the glucosylation of flavonoids. The formation of composite particles may be an alternative strategy to enhanced solubility and may lead to radioprotection.

Furthermore, lambda DNA is a double-stranded DNA with 48,502 base pairs. This size is separable with simple gel electrophoresis and is readily obsevable miscroscopically when using the molecular combing method. Therefore, Lambda DNA is a good test subject for screening purposes such as that 
identified in the present study. Moreover, DNA DSBs lead to serious biological consequences, including mutations, carcinogenesis, or apoptosis (29). We used two assays to measure DSBs. The gel electrophoresis assay is convenient and can be applied with multiple samples. On the other hand, the molecular combing method is time-consuming and labor intense. However, in the molecular combing method one can visualize individual DNA DSB in a single molecule. DNA fragment size can be directly measured in this method. Therefore, gel electrophoresis is suitable for screening purposes and detailed analysis and confirmation can be achieved using the molecular combing method.

However, there were some potential pitfalls in our procedures. The molecular combing method may have some difficulties for reproducibility without careful sample handling. It is possible that different sizes of Lambda DNA do not attach onto cover glass equally. We found that the length of the Lambda DNA decreased sharply with vigorous pipetting, as previously reported (30). We used a $60 \times 24 \mathrm{~mm}$ coverslip as previously reported (24) after we tested several different sizes of coverslips. Interestingly, 55×24 and $45 \times 24 \mathrm{~mm}$ coverslips from the same company did not produce a better quality of Lambda DNA stretch. It is not known why Lambda DNA stretched well at $60 \times 24 \mathrm{~mm}$, while other size slides did not, but it may be associated with the different surface tension.

In conclusion, antioxidant and scavenging capacity are well related to DNA DSB formation reduction. Glucosylation affected antioxidant and free radical scavenging abilities to some degree. Although some flavonoids, especially glucosylated ones such as MG-Rutin and MO-Rutin, have good protection for in vitro double-stranded DNA, it may be limited to in vitro radioprotective effects. Further in vitro cell culture studies and most importantly in vivo studies are required to prove glucosyl flavonoids are good radioprotectors.

\section{Acknowledgements}

The present study was partially supported by the Colorado State University Start Up Fund (TAK), the Collaborative Research Fund from Toyo Sugar Refining Co., Ltd. (MU), and Contract Research Fund from Toyo Sugar Refining Co., Ltd. (TAK).

\section{References}

1. Harman D: Aging: a theory based on free radical and radiation chemistry. J Gerontol 11: 298-300, 1956.

2. Ward JF: DNA damage produced by ionizing radiation in mammalian cells: identities, mechanisms of formation, and reparability. Prog Nucleic Acid Res Mol Biol 35: 95-125, 1988.

3. Lobo V, Patil A, Phatak A and Chandra N: Free radicals, antioxidants and functional foods: impact on human health. Pharmacogn Rev 4: 118-126, 2010.

4. Best BP: Nuclear DNA damage as a direct cause of aging. Rejuvenation Res 12: 199-208, 2009.

5. Andersson DI, Slechta ES and Roth JR: Evidence that gene amplification underlies adaptive mutability of the bacterial lac operon. Science 282: 1133-1135, 1998.

6. Baumann P and West SC: Role of the human RAD51 protein in homologous recombination and double-stranded-break repair. Trends Biochem Sci 23: 247-251, 1998.

7. Kumar S and Pandey AK: Chemistry and biological activities of flavonoids: an overview. ScientificWorldJournal 2013: 162750,2013.

8. Harborne JB and Paxman GJ: Genetics of anthocyanin product in the radish. Hered Edinb 19: 505-506, 1964.
9. Thompson M, Williams CR and Elliot GE: Stability of flavonoid complexes of copper(II) and flavonoid antioxidant activity. Anal Chim Acta 85: 375-381, 1976.

10. Svobodová A, Psotová J and Walterová D: Natural phenolics in the prevention of UV-induced skin damage. A review. Biomed Pap Med Fac Univ Palacky Olomouc Czech Repub 147: 137-145, 2003.

11. Rahman K: Studies on free radicals, antioxidants, and co-factors. Clin Interv Aging 2: 219-236, 2007.

12. Plumb GW, De Pascual-Teresa S, Santos-Buelga C, Cheynier V and Williamson G: Antioxidant properties of catechins and proanthocyanidins: effect of polymerisation, galloylation and glycosylation. Free Radic Res 29: 351-358, 1998.

13. Shimoi K, Yoshizumi K, Kido T, Usui Y and Yumoto T: Absorption and urinary excretion of Quercetin, Rutin, and alphaG-Rutin, a water soluble flavonoid, in rats. J Agric Food Chem 51: 2785-2789, 2003.

14. Engen A, Maeda J, Wozniak DE, Brents CA, Bell JJ, Uesaka M, Aizawa $\mathrm{Y}$ and Kato TA: Induction of cytotoxic and genotoxic responses by natural and novel Quercetin glycosides. Mutat Res Genet Toxicol Environ Mutagen 784-785: 15-22, 2015.

15. Maeda J,RoybalEJ,Brents CA,Uesaka M, Aizawa Y and Kato TA: Natural and glucosyl flavonoids inhibit poly(ADP-ribose) polymerase activity and induce synthetic lethality in BRCA mutant cells. Oncol Rep 31: 551-556, 2014.

16. Sunada S, Fujisawa H, Cartwright IM, Maeda J, Brents CA, Mizuno K, Aizawa Y,Kato TA and Uesaka M: Monoglucosyl-Rutin as a potential radioprotector in mammalian cells. Mol Med Rep 10: 10-14, 2014.

17. Chandrasekharan DK, Kagiya TV and Nair CKK: Radiation protection by 6-palmitoyl ascorbic acid-2-glucoside: studies on DNA damage in vitro, ex vivo, in vivo and oxidative stress in vivo. J Radiat Res (Tokyo) 50: 203-212, 2009.

18. Kobashigawa S, Kashino G, Suzuki K, Yamashita S and Mori H: Ionizing radiation-induced cell death is partly caused by increase of mitochondrial reactive oxygen species in normal human fibroblast cells. Radiat Res 183: 455-464, 2015.

19. Kemp LM, Sedgwick SG and Jeggo PA: X-ray sensitive mutants of Chinese hamster ovary cells defective in double-strand break rejoining. Mutat Res 132: 189-196, 1984.

20. Wlodek D and Olive PL: Physical basis for detection of DNA double-strand breaks using neutral filter elution. Radiat Res 124: 326-333, 1990.

21. Olive PL, Wlodek D and Banáth JP: DNA double-strand breaks measured in individual cells subjected to gel electrophoresis. Cancer Res 51: 4671-4676, 1991.

22. Kato TA, Okayasu R, Jeggo PA and Fujimori A: ASPM influences DNA double-strand break repair and represents a potential target for radiotherapy. Int J Radiat Biol 87: 1189-1195, 2011.

23. Fujii Y, Genet MD, Roybal EJ, Kubota N, Okayasu R, Miyagawa K, Fujimori A and Kato TA: Comparison of the bromodeoxyuridine-mediated sensitization effects between low-LET and high-LET ionizing radiation on DNA double-strand breaks. Oncol Rep 29: 2133-2139, 2013.

24. Oshige M, Yamaguchi K, Matsuura S, Kurita H, Mizuno A and Katsura S: A new DNA combing method for biochemical analysis. Anal Biochem 400: 145-147, 2010.

25. Karioti A, Hadjipavlou-Litina D, Mensah ML, Fleischer TC and Skaltsa H: Composition and antioxidant activity of the essential oils of Xylopia aethiopica (Dun) A. Rich. (Annonaceae) leaves, stem bark, root bark, and fresh and dried fruits, growing in Ghana. J Agric Food Chem 52: 8094-8098, 2004.

26. Bansal V, Sharma A, Ghanshyam C and Singla ML: Coupling of chromatographic analyses with pretreatment for the determination of bioactive compounds in Emblica officinalis juice. Anal Methods 6: 410-418, 2014.

27. Solimani R: The flavonols Quercetin, Rutin and morin in DNA solution: UV-vis dichroic (and mid-infrared) analysis explain the possible association between the biopolymer and a nucleophilic vegetable-dye. Biochim Biophys Acta 1336: 281-294, 1997.

28. Fujimori M, Kadota K, Shimono K, Shirakawa Y, Sato H and Tozuka Y: Enhanced solubility of Quercetin by forming composite particles with transglycosylated materials. J Food Eng 149: 248-254, 2015.

29. Khanna KK and Jackson SP: DNA double-strand breaks: signaling, repair and the cancer connection. Nat Genet 27: 247-254, 2001.

30. Yoo HB, Lim HM, Yang I, Kim SK and Park SR: Flow cytometric investigation on degradation of macro-DNA by common laboratory manipulations. J Biophys Chem 2: 102-111, 2011. 